\title{
Different brain networks mediate task performance in normal aging and $A D$ \\ Defining compensation
}

\author{
Y. Stern, PhD; J.R. Moeller, PhD; K.E. Anderson, MD; B. Luber, PhD; N.R. Zubin, BA; A.A. DiMauro, BA; \\ A. Park, BA; C.E. Campbell, MA; K. Marder, MD, MPH; K. Bell, MD; R. Van Heertum, MD; \\ and H.A. Sackeim, PhD
}

\begin{abstract}
Article abstract-Objective: To determine whether the pathologic mechanisms of $\mathrm{AD}$ alter the brain networks subserving performance of a verbal recognition task. Background: Functional imaging studies comparing task-related activation in $\mathrm{AD}$ patients and controls generally have not used network analysis and have not controlled for task difficulty. Methods: $\mathrm{H}_{2}{ }^{15} \mathrm{O}$ PET was used to measure regional cerebral blood flow in 14 patients and 11 healthy elders during the performance of a serial verbal recognition task under two conditions: low demand, with study list size (SLS) equal to one; and titrated demand, with SLS adjusted so that each subject recognized words at 75\% accuracy. The Scaled Subprofile Model was used to identify networks of regionally covarying activity across these task conditions. Results: In the elders, higher SLS was associated with the recruitment of a network of brain areas involving left anterior cingulate and anterior insula $\left(R^{2}=\right.$ $0.94 ; p<0.0001)$. Three patients also expressed this network. In the remaining patients, higher SLS was associated with the recruitment of an alternate network consisting of left posterior temporal cortex, calcarine cortex, posterior cingulate, and the vermis $\left(R^{2}=0.81, p<0.001\right)$. Expression of this network was unrelated to SLS in the elders and more intact AD patients. Conclusions: The patients' use of the alternate network may indicate compensation for processing deficits. The transition from the normal to the alternate network may indicate a point where brain disease has irreversibly altered brain function and thus may have important implications for therapeutic intervention.
\end{abstract}

NEUROLOGY 2000;55:1291-1297

After brain injury, the same brain networks that normally mediate performance of a cognitive task may continue to operate at reduced efficiency, or the normal networks may be replaced by alternate networks to compensate for the loss of normal function. Recent research has used functional neuroimaging to address this issue in aged adults, ${ }^{1}$ recovering stroke patients, ${ }^{2}$ and in patients with $\mathrm{AD}{ }^{3}$ In the current study, we determined the brain network underlying the performance of a continuous verbal recognition task in healthy elders. We then determined the degree to which network function is altered by $\mathrm{AD}$.

More extensive recruitment of brain areas during task performance has been noted in $\mathrm{AD}$ patients than in age-matched controls and has been interpreted as patients' attempts to compensate for disease by using alternate cognitive processes. ${ }^{4-6}$ However, studies of healthy individuals demonstrate similar alterations in activation as tasks are made more difficult. ${ }^{7-10}$ Because most tasks are more difficult for $\mathrm{AD}$ patients than healthy elders, it is important to determine whether observed changes in activation in $\mathrm{AD}$ simply represent modulation in the use of normal networks. In the current study, we matched task difficulty for each study participant. We hypothesized that some AD patients would continue to use the same networks as healthy elders, whereas others would use an alternate network.

Methods. Subjects. Eleven healthy elderly controls and 14 patients who met research criteria for probable $\mathrm{AD}^{11}$ participated in this $\mathrm{H}_{2}{ }^{15} \mathrm{O}$ PET study. Demographic and clinical variables are summarized in the table. Patients were slightly younger than the healthy elders $(t=1.2$, not significant) and had comparable years of education. Patient test scores were consistent with mild dementia: Modified Mini-Mental State Examination score, Wechsler Adult Intelligence-Revised Scale Full Scale IQ, and Wechsler Memory Scale Memory Quotient were significantly lower in patients than controls. Informed written consent was obtained after the nature and risks of the study were explained.

Activation task. The activation task was initiated 1-minute before the start of the scan and continued throughout the scan period. The continuous performance verbal recognition test consisted of serial presentation of one or more single words followed by a series of recognition

From the Departments of Neurology (Drs. Stern, Marder, and Bell), Psychiatry (Drs. Stern, Moeller, Anderson, Luber, Marder, and Sackeim and C.E. Campbell), and Radiology (Dr. Van Heertum) and the Cognitive Neuroscience Division of the Sergievsky Center (Drs. Stern and Anderson and N.R. Zubin, A.A. DiMauro, and A. Park), College of Physicians and Surgeons of Columbia University; The Alzheimer's Disease Research Center in the City of New York (Drs. Stern, Anderson, Marder, and Bell); and the Department of Biological Psychiatry (Drs. Stern, Moeller, Luber, and Sackeim), New York State Psychiatric Institute, New York, NY.

Supported by federal grants AG16671, AG08702, RR00645, and the Higginbottom and Milstein Foundations.

Received March 22, 2000. Accepted in final form July 20, 2000.

Address correspondence and reprint requests to Dr. Stern, Sergievsky Center, 630 W 168th Street, New York, NY 10032; e-mail: ys11@columbia.edu 
Table Demographic and clinical information for the two subject groups

\begin{tabular}{lcc}
\hline Characteristics & $\begin{array}{c}\text { Normal elders } \\
(\mathrm{n}=11)\end{array}$ & $\begin{array}{c}\text { AD patients } \\
(\mathrm{n}=14)\end{array}$ \\
\hline Age & $73.2(6.6)$ & $67.8(12.5)$ \\
Education & $16.2(1.8)$ & $15.5(2.9)$ \\
Modified mMMS & $53.3(3.4)$ & $45.5(4.2)$ \\
WAIS-R Full Scale IQ & $119.3(13.7)$ & $94.4(13.5)$ \\
Wechsler Memory Scale MQ & $134.6(11.9)$ & $91.9(12.6)$ \\
\hline
\end{tabular}

Values are mean (SD).

mMMS $=$ Mini-Mental State Examination; WAIS-R $=$ Wechsler Adult Intelligence Scale-Revised; MQ = Memory Quotient.

probes. Individuals viewed word stimuli on an overhead monochrome monitor while lying in a supine position. There was an alternating sequence of study and test blocks. Test probes were distinguished from study items by a circumscribed rectangular frame. Study words were displayed for a 2-second duration with a 133-millisecond interstimulus interval. A 500-millisecond delay occurred at the transition to test trials, after which test probes were displayed. A subject was instructed to make a "new" or "old" response on each test trial by pressing one of two microswitches with the left or right thumb. Accuracy was emphasized over speed. A new test probe was displayed immediately after a button press. A 4-second response time limit was imposed. A 500-millisecond delay occurred at the transition to the next study phase. "New" and "old" test probes occurred with a frequency of $50 \%$. Test probes were pseudorandomized so that no more than four consecutive trials required the same response. Study words and their associated test probes were matched for letter length, word frequency, part of speech, and imagability. All word stimuli in study and test blocks were commonly used words with frequencies of once per million or greater (Kucera and Frances norms). The semantic similarity among word stimuli was controlled across study and test blocks by separating semantically related words by 10 or more unrelated intervening words.

Two versions of the recognition task were used. In the low-demand condition, the study list size (SLS) was one (i.e., one study word followed by one recognition probe). The titrated demand condition used a predetermined SLS where subject's recognition accuracy was $75 \%$. One day before the PET scan, participants received task instructions and two 20-minute practice sessions. During practice, the particular SLS value at which a subject achieved $75 \%$ accuracy was determined using a staircase method of SLS adjustment. This SLS value then was used in all blocks of the titrated demand condition on the day of the scan. To ensure adequate sampling of brain activity in both study and test trials, SLS was limited to 19 words, making it necessary to exclude healthy elders whose recognition accuracy was greater than $75 \%$ at that SLS cutoff value. In this way, task difficulty was equated across healthy controls and patients.

PET scan acquisition and processing. Scans were separated by 20 minutes and obtained in the following order: rest, low demand, titrated demand, titrated demand, low demand, rest. Regional cerebral blood flow ( $\mathrm{rCBF}$ ) values were determined using the autoradiographic method. ${ }^{12}$ For each scan, a bolus of $30 \mathrm{mCi}$ of IV $\mathrm{H}_{2}{ }^{15} \mathrm{O}$ was injected 60 seconds after initiation of one of the memory tasks. Using a Siemens EXACT 47 PET camera (Munich, Germany), three 30-second scan frames were acquired in twodimensional mode beginning 20 seconds after tracer administration. After measured attenuation correction (15-minute transmission scan) and reconstruction by filtered backprojection, image resolution was $10 \mathrm{~mm}$ full width, half maximum. Arterial blood sampling was not conducted.

Radioactive counts were used to estimate relative $\mathrm{rCBF}$ values. ${ }^{13}$ Using modules from the Statistical Parametric Mapping program (SPM96), each subject's scans were realigned to each other and normalized into standardized Talairach space. $\mathrm{MED}_{\mathrm{X}}$ (Sensor Systems, Inc, Sterling, VA) software then was used to overlay a standardized three-dimensional region of interest (ROI) template on the scan slices. This template was drawn on the SPM96 standard MRI (which conforms to Tailarach space) and defined 21 ROI per hemisphere, plus one midline ROI, comprising major cortical and subcortical regions (figure 1). To prepare the data for Scaled Subprofile Modeling analyses, an automated segmentation-like method, averaging the pixels with the $20 \%$ highest activity within the ROI, was applied to every ROI to distinguish gray matter from white matter and CSF, and white matter from CSF, as well as to correct for atrophy.

Topography analyses. To identify the brain networks underlying task performance, we analyzed the ROI activation data using the Scaled Subprofile Model (SSM). ${ }^{14-16}$ SSM is a form of principal component analysis that extracts one or more topographies, which consist of regional weights that are independent of a global mean effect and that represent covarying patterns of $\mathrm{rCBF}$. Each of these covariance patterns potentially represents a pattern of functional interactions among specific brain regions, that is, a brain network. Each brain region has an associated weighting that expresses the degree to which it participates in the specific topography, either by increasing or by decreasing $\mathrm{rCBF}$. SSM was applied to the change in $\mathrm{rCBF}$ from the low to the titrated demand condition. The topography was assumed to represent a network of brain areas engaged by the task, and a change in the degree of expression of a topography across the two conditions was assumed to be related to the increased demand presented by the titrated condition. A subject scaling factor (SSF) also was calculated for each subject, which represents the degree to which the subject expresses the particular topography. The SSF is offset relative to the mean subject expression of network activity. That is, subjects with midrange expression exhibit an SSF value near zero. Subjects with higher scores show a relative increase in flow in some areas and decreases in others, whereas the opposite pattern of flow increases and decreases is seen in subjects with negative scores.

To evaluate the patterns of activation associated with performing the word recognition task, our strategy was to separately identify the networks underlying task performance in the normal elderly and AD groups. SSM was applied to the titrated minus low-demand subtraction data. This approach identifies networks whose expression changed across these two conditions. The SSF scores obtained from the SSM analysis subsequently were corre- 


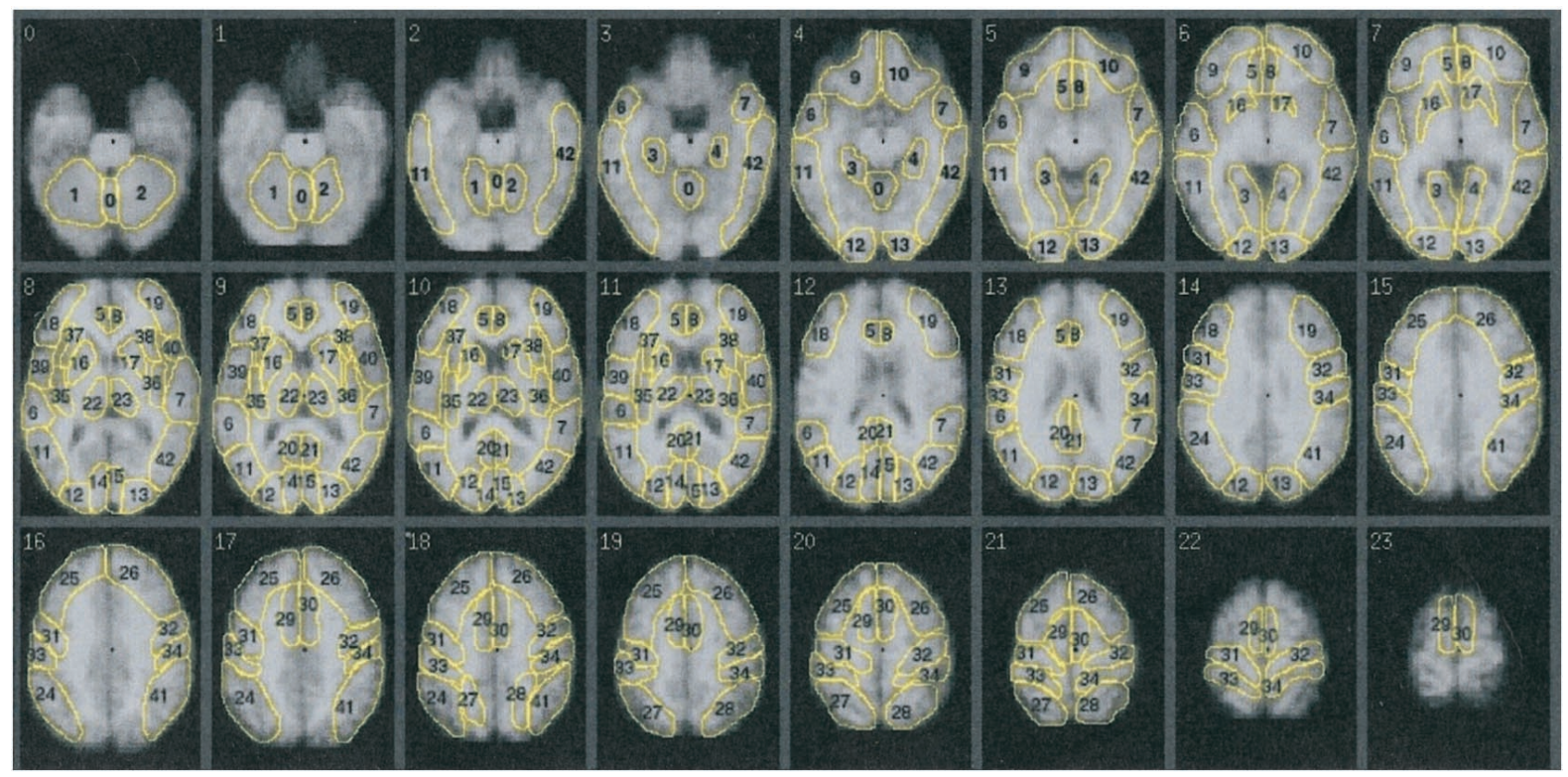

Figure 1. Regions of interest (ROI) included in the current analyses. Except for the vermis, values in parentheses indicate the number of ROI for right and left. Vermis (0), cerebellum (1,2), hippocampus (3,4), anterior cingulate $(5,8)$, superior temporal (6,7), orbital frontal (9,10), parietal Temporal (11,42), association cortex (12,13), calcarin (14,15), basal ganglia $(16,17)$, dorsolateral prefrontal $(18,19)$, posterior cingulate $(20,21)$, thalamus $(22,23)$, inferior parietal $(24,41)$, superior frontal (25,26), superior parietal $(27,28)$, supplementary motor cortex $(29,30)$, premotor cortex $(31,32)$, primary sensory cortex $(33,34)$, posterior insula $(35,36)$, anterior insula $(37,38)$, and operculum $(39,40)$.

lated with a behavioral index of performance, SLS in the titrated demand condition. A significant correlation provides independent evidence that the identified regions operate as a network that mediates task performance.

Once a topography is identified in the subtraction images of a specific group, the degree to which the pattern is expressed in the data of any other subject can be calculated. ${ }^{15,17}$ The computation of a subject's score for a given topography from individual subtraction images was performed on standardized ROI data by using an automated algorithm (Topographic Profile Rating). The Topographic Profile Rating was performed, blind to all subjectidentifying information including group classifications, to obtain patients' expression of the healthy elders' topography and elders' expression of the patients' topography.

Results. Behavioral results. The mean SLS in the titrated demand condition was $13.9 \pm 1.5$ in the controls and $7.4 \pm 4.2$ in the patients $(t=4.89, p<0.001)$. SLS ranged from 12 to 17 in the healthy elders and from 2 to 15 in the AD patients. For 11 of the patients, the SLS was below 10, whereas 3 had an SLS of 11 or higher. During the scans, both groups performed the low-demand task at approximately $95 \%$ accuracy and the titrated demand task at $75 \%$ accuracy.

Physiologic results. Healthy elders' network. The SSM analysis identified a topography whose differential expression (i.e., individual differences in SSF values) correlated with the SLS attained in the titrated demand condition $\left(R^{2}=0.94 ; p<0.0001\right.$; figure 2A). Achieving higher SLS was associated with increased CBF in the left anterior cingulate and anterior insula and decreased $\mathrm{CBF}$ in the left basal ganglia (figure $3 \mathrm{~A}$ ).

We calculated the degree to which the $\mathrm{AD}$ patients expressed the normal elders' topography. As depicted in fig- ure $2 \mathrm{~B}$, expression of the normal topography did not relate to SLS in most patients. For most, the SSF for expression of this topography was close to zero, indicating that these patients did not express this activation pattern. The lack of expression of the healthy elders' topography did not simply result from reduced ability to recruit $\mathrm{CBF}$ in the constituent brain regions; $t$-tests demonstrated that the standardized $\mathrm{CBF}$ values in each of these regions during the low or titrated demand conditions did not differ significantly across the two groups ( $p>0.10$ for all comparisons, figure 4). Thus, the difference between the two groups resulted from differences in how activation covaried among the implicated brain regions.

In contrast, the data for the three $\mathrm{AD}$ patients who had achieved relatively high SLS (i.e., >10) fit well on the regression line between the healthy elders' expression of topography and SLS (see figure 2B), suggesting that they were effectively using the healthy elders' brain network.

$\mathrm{AD}$ patients' network. In a separate analysis of the PET activation data in the $11 \mathrm{AD}$ patients with lower SLS (i.e., SLS <10), the SSM analysis identified a different topography whose expression by these patients also correlated with SLS in the titrated demand condition $\left(R^{2}=\right.$ $0.81, p<0.001$; see figure 2C). The three patients with higher SLS were excluded from this analysis because they appeared to be manifesting the healthy elders' brain network, and the intent of this analysis was to obtain an unbiased estimate of the alternate, disease-related topography. Within this $\mathrm{AD}$ subgroup, attaining a larger SLS was associated with increased CBF in left posterior temporal cortex, calcarine cortex, posterior cingulate, and the vermis (see figure 3B).

We also calculated the degree to which the healthy elders and the three high-SLS AD patients expressed the topography observed in poor performing $\mathrm{AD}$ patients. As 


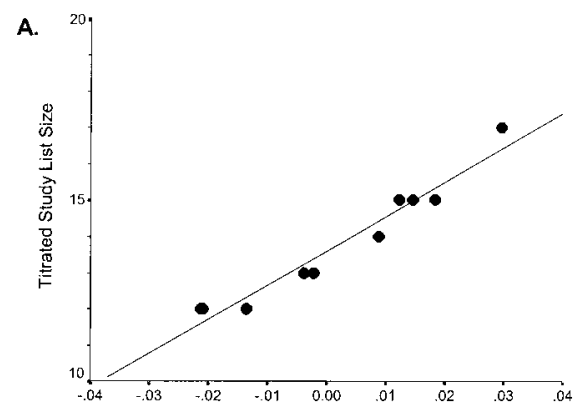

SSF: Healthy Elders' Topography

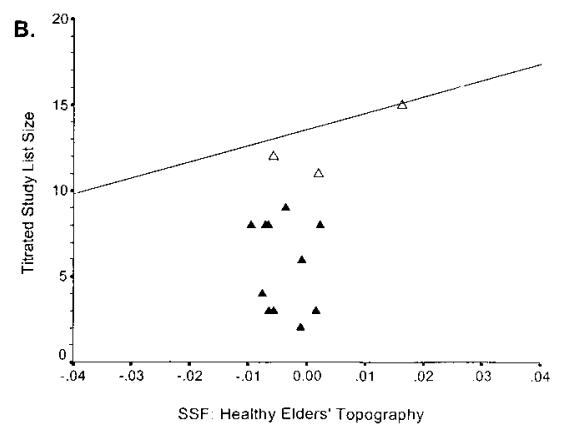

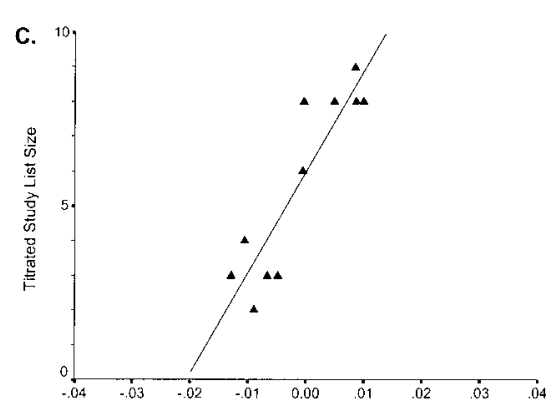

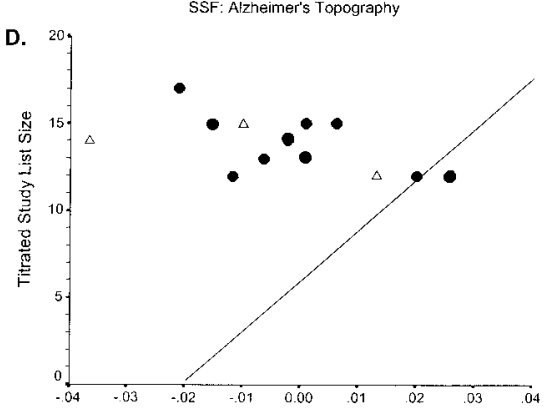

SSF: Alzheimer's Topography
Figure 2. Scatter plots of the study list size (SLS) attained in the titrated demand condition and its predictor, subjects' expression of a topography as quantified by the Subject Scaling Factor (SSF). In all scatter graphs, healthy elderly individuals are represented with closed circles, $A D$ patients with low SLS $(<10)$ with closed triangles, and patients with high SLS with open triangles. (A) Correlation between healthy elders' SLS and expression of their topography. (B) Scatter plot of the relation between SLS in high-and low$S L S A D$ patients and expression of the healthy elder topography. The regression line of figure $1 \mathrm{~A}$ is reproduced for reference. Notice that the data for the high SLS AD patients fall along that regression line, suggesting that these individuals are expressing and using this network in the same way as healthy controls. (C) Correlation between $S L S$ in low-SLS AD patients and expression of their topography. (D)

Scatter plot of high-SLS AD patients' and healthy elders' SLS and expression of the AD patient topography. The regression line of figure $1 C$ is reproduced for reference. Notice that the data for the healthy elders and high-SLS AD patients do not fall along that regression line, suggesting that their expression of this network is not related to task performance.

seen in figure $2 \mathrm{D}$, these individuals did not express the topography in a manner that predicted SLS. The absence of this relationship in the healthy elders did not result simply from differential recruitment of CBF in the constituent brain regions; $t$-tests demonstrated that the standardized CBF values in each of these regions did not differ significantly between the two groups $(p>0.10$ for all comparisons; see figure 4). Thus, the difference between the two groups resulted from differences in how activation covaried among the implicated brain regions.

Discussion. In cognitive neuroscience, the goal of neuroimaging often is to associate a single cognitive process with activity in a specific functional brain pathway. However, the brain damage that occurs in neurodegenerative diseases like $\mathrm{AD}$ does not always follow the anatomic demarcations of any specific information processing pathway. In early $\mathrm{AD}$, corticocortical connections are affected throughout the association cortex, and some cognitive changes can result from loss of connectivity between distributed neural systems that are required to perform a task. The connections affected are likely to support both attentional processes and working memory networks that mediate a variety cognitive functions. ${ }^{18}$ Similarly, these pathways support declarative memory, which is not a unitary process but involves the operation of several potentially independent but interactive networks. ${ }^{19}$

Our continuous recognition task is likely to make demands on networks mediating encoding, storage,
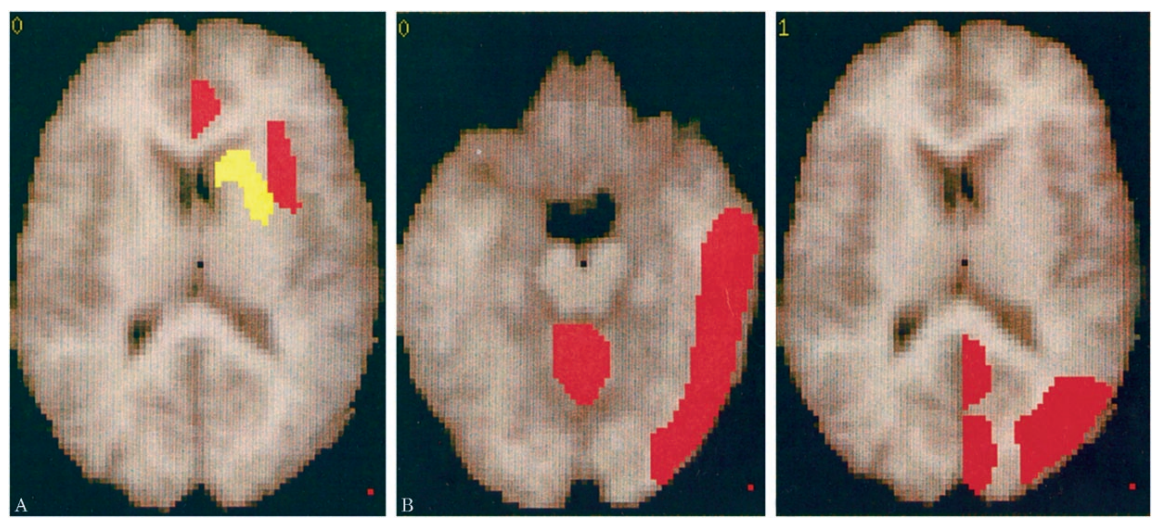

Figure 3. Covariance patterns (topographies) expressed by the healthy elderly and $A D$ subjects. Within each group, differential expression of these topographies correlates with study list size (SLS) on the activation task. Weights for each region's participation in the topography have been overlaid on standard, Tailarach transformed axial MRI sections, with positive weights indicated in red and negative weights indicated in blue. (A) The healthy elders' topography. Higher SLS was associated with increased activation in left anterior cingulate and anterior insula and decreased activation of the left basal ganglia. (B) AD topography. Higher SLS was associated with the increased activation of the left posterior temporal cortex, calcarine cortex, posterior cingulate, and the vermis. 


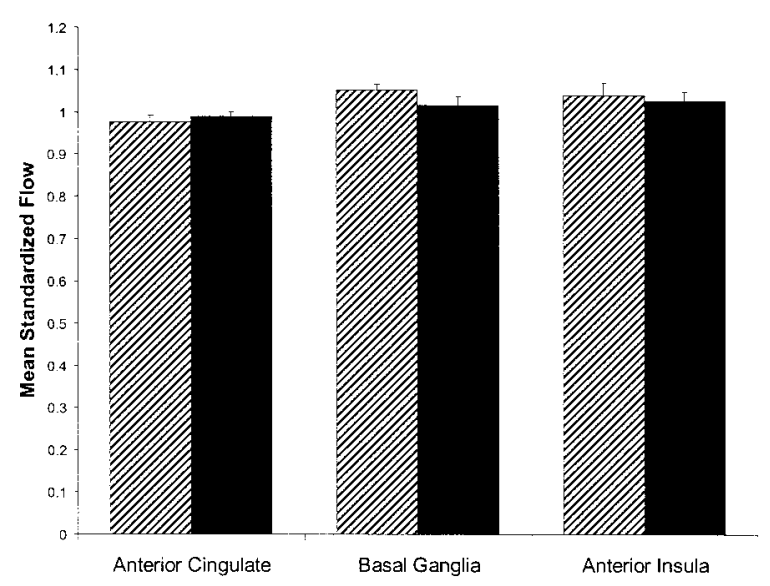

B

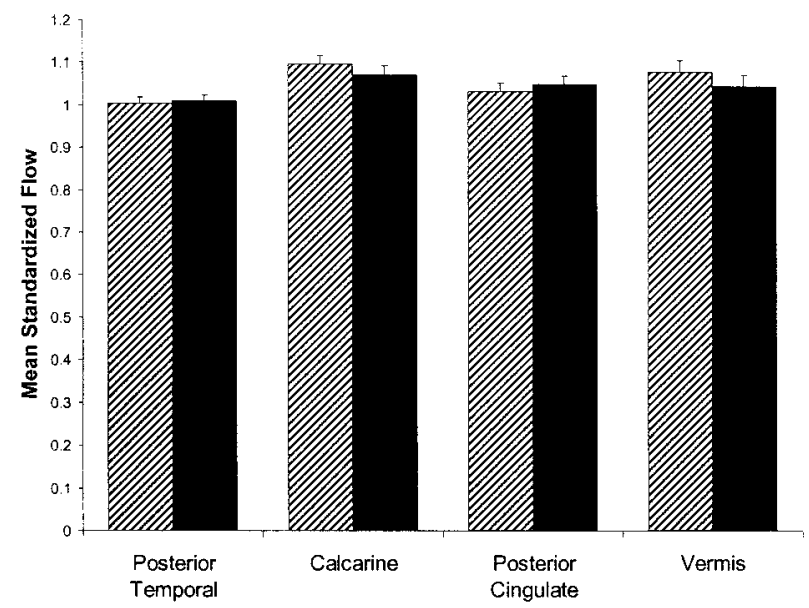

Figure 4. Standardized flow values for regions of interest that participate in the normal topography $(A)$ and the $A D$ topography (B). Data are presented for the 11 healthy elders (solid black) and the $11 \mathrm{AD}$ patients (hatch marks) with study list sizes $<10$. Flow values in the titrated demand condition are presented for each region of interest. Flow values did not differ in $A D$ patients and healthy elders in any region.

and retrieval, as well as aspects of attention and working memory. The task was designed to have diverse information processing and memory demands. Thus, normal performance should reflect interactions among several brain networks. In $\mathrm{AD}$, we might expect to see the loss of interactions among some brain areas, and, to the degree that $\mathrm{AD}$ patients can compensate for deficits, new interactions can arise that may involve different brain regions. The analytic methods used in the current study were designed to identify the brain networks engaged by healthy elders during task performance and then to determine whether these networks were affected by $\mathrm{AD}$.

In our analysis, we also distinguished between the use of alternate brain networks and the normal variability in performance caused by individual differences. Our analytic approach accounted for the fact that the relative utilization of brain regions in a network may vary across individuals, even when disease is not present. Thus, we could determine whether the network engaged by the $\mathrm{AD}$ patients was within this normal range of variability. An important design feature was the careful control of task difficulty. By matching task difficulty across subjects, we ensured that differences in the utilization of a network did not simply result from differential task demand. In essence, the titrated demand task required that each subject optimally engage the network that mediated task performance. More typically, the task itself is matched across subjects, and difficulty is allowed to vary. The latter approach initially may seem more attractive because the task administered to all subjects appears to be the same; however, the actual difficulty demands it places on each subject can vary widely.

Our findings suggest that healthy elders engaged a specific brain network when performing a continuous verbal recognition task. This contention is bolstered by the observation that differential expression of this network was strongly related to the SLS attained by elders in the titrated demand condition. Three $\mathrm{AD}$ patients also expressed this network in the same manner, suggesting that they were performing the task similarly to healthy elders. We hypothesize that $\mathrm{AD}$ had not yet disrupted the normal network in these patients.

Consideration of the brain regions that participated in the network used by the healthy elders suggests that this network is involved in transforming the recognition task from one which requires attentional resources to a more automated task. Higher SLS was associated with relatively increased activation of the left anterior cingulate and insula. In previous PET studies of memory and linguistic functions involving visually presented words, activation of the anterior cingulate has been associated with attentional demands involving response selection..$^{20-26}$ Insular cortex activations have been associated with motor planning activity and overlearned or automatic task performance, ${ }^{27}$ as well as with top-down attentional control. ${ }^{28}$ The basal ganglia, also a part of this network, have been implicated in aspects of cognitive/motor coordination and the sequencing of task demands. ${ }^{29-32}$ In summary, this network appears to allow subjects to work with increased SLS by modulating attentional resources and mediating sequential and response selection demands.

Performance (i.e., SLS) across the healthy elders and the three $\mathrm{AD}$ patients was variable, but it was related to modulation in the expression of the same network. Individual differences in performance may represent differences in the ability to effectively recruit this network. Individuals who can recruit this normal network to a greater degree might be able to continue to do so more effectively in the face of significant brain injury, but this possibility could not be assessed in the current study.

For most of the $\mathrm{AD}$ patients, task performance was associated with activation of a different network 
than that used by controls. In response to the increased load of the titrated demand task, the patients recruited a network of posterior areas that are involved in the initial processing and encoding of the word stimuli. Left posterior temporal cortex typically is activated in tasks that involve orthographic processing of words, ${ }^{33-35}$ and calcarine cortex is activated with any patterned stimuli. Posterior cingulate cortex has been activated routinely in tasks requiring semantic associations with words. ${ }^{34,36}$ The vermis has been activated in tasks using visual word and letter stimuli. ${ }^{34,37}$ The contrast of the low and titrated demand conditions would not be expected to show memory-specific activation (e.g., activation of perihippocampal regions) because both conditions require encoding and retrieval. However, this posterior, perceptual processing network could be working in tandem with memory-related areas to mediate the increase in the encoding and retrieval demands across the two task conditions.

The alternate network did not mediate task performance in the healthy elders and the three better performing patients, as indicated by the lack of correlation between subjects' SLS and the SSF scores, which measure their expression of this network. Still, as seen in figure 2D, this network was expressed by controls during task performance, suggesting that this perceptual processing network was engaged but was not responsible for the elders' ability to work with larger study lists. This observation is consistent with the notion that both brain networks were operating concurrently in the healthy elders.

Several other functional imaging studies have noted more extensive recruitment of brain areas in $\mathrm{AD}$ patients than in age-matched controls, which have been interpreted as the patients' attempts to compensate for disease by using alternate cognitive processes. $^{4-6}$ Differences in the relative difficulty of a task across groups also can result in more extensive recruitment. ${ }^{7}$ In contrast to these other studies, making the current task more difficult for the normal elders could not have produced a pattern of activation like that seen in the patients because the $A D$ patients had a more restricted pattern of activation and were unable to use the same normal network as controls.

Some methods of modeling brain activation may be more useful than others for identifying brain networks and for determining whether experimental groups are expressing the same or different networks. A standard approach is to make betweencondition and between-group comparisons by contrasting mean activation images on a pixel-bypixel basis. An alternate approach, used here, was to compare changes in patterns of covariance across conditions. The latter approach may be more successful in defining functional networks. For example, one study used the more standard approach and found between-group and condition differences, suggesting that, while performing a verbal memory task, $\mathrm{AD}$ patients used more extensive brain areas than controls. ${ }^{5}$ An alternate analysis of the same data using a factor analytic approach suggests that the functional organization of the verbal memory system was comparable in both groups. ${ }^{38}$ In the current study, simple comparison of ROI did not identify differences between groups, whereas SSM identified these ROI as key participants in networks associated with task performance. The use of ROI and the automated segmentation-like method described in Methods also may help to overcome image processing difficulties that can result from the cerebral atrophy common in aging and $\mathrm{AD}$.

Whether the patients' use of the alternate network represents compensation is a matter of definition. The alternate network was used by patients in the place of the normal network to mediate task demands, suggesting that its use was compensatory. However, this network also was activated by the healthy elders, indicating that it was not unique to the patients. If the term compensation is reserved for the use of a novel network that emerges in response to disease, then the alternate network does not meet this criterion. On the other hand, the role played by the alternate network differed in patients and controls in that it appeared to be mediating the ability to achieve larger SLS in the patients but not the elders. This novel use of the network may arise out of the inability to use the standard network and thus may be considered compensation.

These initial findings provide a framework for further investigation of compensation in response to brain disease and of individual differences in task performance. Also, the use of an alternate network may indicate a point at which disease has irreversibly altered brain function. The ability to determine whether a patient expresses a normal or alternate brain network therefore may have implications for remediation or therapeutic intervention, with the transition to an alternate network indicating a point where interventions are less likely to be effective.

\section{References}

1. Grady CL, Maisog JM, Horwitz B, et al. Age-related changes in cortical blood flow activation during visual processing of faces and location. J Neurosci 1994;14:1450-1462.

2. Weiller C, Ramsay SC, Wise RJS, Friston KJ, Frackowiak RSJ. Individual patterns of functional reorganization in the human cerebral cortex after capsular infarction. Ann Neurol 1993;33:181-189.

3. Weder B, Knorr U, Herzog B, Steinmetz H, Seitz RJ. Tactile exploration of shape after subcortical ischaemic infarction studied with PET. Brain 1994;117:593-601.

4. Grady CL, Haxby JV, Horwitz B, et al. Activation of cerebral blood flow during a visuoperceptual task in patients with Alzheimer-type dementia. Neurobiol Aging 1993;14:35-44.

5. Becker JT, Mintun MA, Aleva K, et al. Compensatory reallocation of brain resources supporting verbal episodic memory in Alzheimer's disease. Neurology 1996;46:692-700.

6. Backman L, Andersson JL, Nyberg L, et al. Brain regions associated with episodic retrieval in normal aging and Alzheimer's disease. Neurology 1999;52:1861-1870.

7. Gur RC, Gur RE, Skolnick BE, et al. Effects of task difficulty on regional cerebral blood flow: relationships with anxiety and performance. Psychophysiology 1988;25:392-399. 
8. Rypma B, Prabhakaran V, Desmond JE, Glover GH, Gabrieli JD. Load-dependent roles of frontal brain regions in the maintenance of working memory. Neuroimage 1999;9:216-226.

9. Grasby PM, Frith CD, Friston KJ, et al. A graded task approach to functional mapping of areas implicated in auditoryverbal memory. Brain 1994;117:1271-1282.

10. Grady CL, Horwitz B, Pietrini P, et al. The effect of task difficulty on cerebral blood flow during perceptual matching of faces. Hum Brain Mapping 1996;4:227-239.

11. McKhann G, Drachman D, Folstein M, et al. Clinical diagnosis of Alzheimer's disease: report of the NINCDS-ADRDA Work Group. Neurology 1984;34:939-944.

12. Herscovitch P, Markham J, Raichle ME. Brain blood flow measured with intravenous H2(15)0. J Nucl Med 1983; 24: 782-789.

13. Fox PT, Mintun MA. Noninvasive functional brain mapping by change-distribution analysis of averaged PET images of $\mathrm{H}_{2}{ }^{15} 0$ tissue activity. J Nucl Med 1989; 30:141-149.

14. Moeller JR, Strother SC, Sidtis JJ, Rottenberg DA. Scaled subprofile model: a statistical approach to the analysis of functional patterns in positron emission tomographic data. J Cereb Blood Flow Metab 1987;7:649-658.

15. Alexander GE, Moeller JR. Application of scaled subprofile modeling to functional imaging in neuropsychiatric disorders: a principal component approach to modeling brain function in disease. Hum Brain Mapping 1994;2:79-94.

16. Moeller JR, Ghez C, Antonini A, et al. Brain networks of motor behavior assessed by principal component analysis. In: Carson R, ed. Quantitative Functional Brain Imaging With Positron Emission Tomography. San Diego: Academic Press, 1998:247-252.

17. Eidelberg D, Moeller JR, Antonini A, et al. Functional brain networks in DYT1 dystonia. Ann Neurol 1998;44:303-312.

18. Parasuraman R, Haxby J. Attention and brain function in Alzheimer's disease: a review. Neuropsychology 1993;7:242272.

19. Moscovitch M. Memory and working with memory: evaluation of a component process model and comparisons with other models. In: Schacter D, Tulving E, eds. Memory Systems 1994. Cambridge, MA: MIT Press, 1994:269-310.

20. Petersen SE, Fox PT, Posner MI, Minton M, Raichle ME. Positron emission tomographic studies of the processing of single words. J Cogn Neurosci 1990;1:153-170.

21. Posner MI, Petersen SE. The attention system of the human brain. Neuroscience 1990;13:25-42.

22. Vogt BA, Finch DM, Olson CR. Functional heterogeneity in cingulate cortex: the anterior executive and posterior evaluative regions. Cereb Cortex 1992;2:435-443.
23. Raichle ME, Fiez JA, Videen TO, et al. Practice-related changes in human brain functional anatomy during nonmotor learning. Cereb Cortex 1994;4:8-26.

24. Kapur S, Tulving E, Cabeza R, et al. The neural correlates of intentional learning of verbal materials: a PET study in humans. Cognitive Brain Res 1996;4:243-249.

25. Petersen SE, Fiez JA. The processing of single words studied with positron emission tomography. Ann Rev Neurosci 1993; 16:509-530.

26. Chertkow H, Murtha S. PET activation and language. Clin Neurosci 1997;4:78-86.

27. Augustine JR. Cicuitry and functional aspects of the insular lobe in primates including humans. Brain Res Rev 1996;22: 229-244.

28. Posner MI, DiGirolamo GJ. Executive attention: conflict, target detection, and cognitive control. In: Parasuraman R, ed. The Attentive Brain. Cambridge, MA: MIT Press, 1998:401-424.

29. Marsden CD, Obeso JA. The functions of the basal ganglia and the paradox of stereotaxic surgery in Parkinson's disease. Brain 1994;117:877-897.

30. Houk JC, Wise SP. Distributed modular architectures linking basal ganglia, cerebellum, and cerebral cortex: Their role in planning and controlling action. Cereb Cortex 1995;5:95-110.

31. Saint-Cyr JA, Taylor AE, Nicholson K. Behavior and the basal ganglia. Adv Neurol 1995;65:1-28.

32. Gray JA. A model of the limbic system and basal ganglia: applications to anxiety and schizophrenia. In: Gazzard B, ed. The Cognitive Neurosciences. Cambridge, MA: MIT Press, 1999.

33. Petersen SE, Fox PT, Posner MI, Minton M, Raichle ME. Positron emission tomographic studies of the cortical anatomy of single-word processing. Nature 1988;383:585-589.

34. Vandenberghe R, Price C, Wise R, Josephs O, Frackowiak RSJ. Functional anatomy of a common semantic system for words and pictures. Nature 1996;383:254-256.

35. Price C, Wise R, Frackowiak RSJ. Demonstrating the implicit processing of visually presented words and pseudowords. Cereb Cortex 1996;6:62-70.

36. Demonet J-F, Chollet F, Ramsay S, et al. The anatomy of phonological and semantic processing in normal subjects. Brain 1992;115:1753-1768.

37. Desmond JE, Gabrieli JD, Wagner AD, Ginier BL, Glover GH. Lobular patterns of cerebellar activation in verbal workingmemory and finger-tapping tasks as revealed by functional MRI. J Neurosci 1997;17:9675-9685.

38. Herbster AN, Nichols T, Wiseman MB, et al. Functional connectivity in auditory-verbal short-term memory in Alzheimer'sdisease. Neuroimage 1996;4:67-77.

\section{Activate your online subscription}

At www.neurology.org, subscribers can now access the full text of the current issue of Neurology and back issues to 1995. Select the "Login instructions" link that is provided on the Help screen. Here you will be guided through a step-by-step activation process.
Neurology online offers:

- Access to journal content in both Adobe Acrobat PDF or HTML formats

- Links to PubMed

- Extensive search capabilities

- Complete online Information for Authors

- Examinations on designated articles for CME credit

- Access to in-depth supplementary scientific data 